\title{
Chemical equilibration of QGP in hadronic collisions
}

\author{
Aleksi Kurkela $^{1,2}$ and Aleksas Mazeliauskas ${ }^{1,3}$ \\ 1 Theoretical Physics Department, CERN, 1211 Genève 23, Switzerland \\ 2 Faculty of Science and Technology, University of Stavanger, 4036 Stavanger, \\ Norway \\ 3 Institut für Theoretische Physik, Universität Heidelberg, 69120 Heidelberg, \\ Germany
}

\begin{abstract}
We performed state-of-the-art QCD effective kinetic theory simulations of chemically equilibrating QGP in longitudinally expanding systems. We find that chemical equilibration takes place after hydrodynamization, but well before local thermalization. By relating the transport properties of QGP and the system size we estimate that hadronic collisions with final state multiplicities $d N_{\mathrm{ch}} / d \eta>10^{2}$ live long enough to reach approximate chemical equilibrium for all collision systems. Therefore we expect the saturation of strangeness enhancement to occur at the same multiplicity in proton-proton, proton-nucleus and nucleus-nucleus collisions.
\end{abstract}

The experimental measurements of the final-state particles in the ultra-relativistic proton-proton, proton-nucleus and nucleus-nucleus collisions at hadron colliders show a qualitative change of hadron chemistry with the increasing particle multiplicity [123]. At central nucleus-nucleus collisions the observed ratios of long-lived hadrons is consistent with chemical equilibrium hadron resonance gas models at temperature $T_{\mathrm{ch}} \approx 155 \mathrm{MeV}$ [4, while the lowest multiplicity protonproton collisions are reproduced by perturbative event generators. One interpretation behind this change is the formation of deconfined state of Quark-Gluon Plasma (QGP), which reaches approximate thermal and chemical equilibrium in the collision fireball, from which the thermal hadron productions ensues. Indeed, the hydrodynamic models of the QGP expansion have been very successful in describing small transverse momentum particle spectra and multi-particle correlations. However the initial state created in high-energy nuclear collisions even locally is far from equilibrium and the study of thermalization has been a very active topic [5. In this work 617 we describe the QGP approach to chemical equilibrium within the framework of QCD effective kinetic theory 8 .

At high energy, weak coupling limit of QCD, the mid-rapidity interaction region is dominated by strong non-equilibrium gluonic fields [5]. The microscopic description to local thermal equilibrium from such initial configuration is described by the "bottom-up" thermalization scenario [9, which was explicitly realized by classical-statistical Yang-Mills [10] and gluonic kinetic theory [1] 
simulations. Here for the first time we use the complete leading order QCD kinetic theory of quarks and gluons $[8$ to simulate the hydrodynamic, chemical and kinetic equilibration of QGP. We solve the coupled Boltzmann equations for quark and gluon distribution functions undergoing homogeneous boost-invariant expansion

$$
\partial_{\tau} f_{g, q}-\frac{p_{z}}{\tau} \partial_{p_{z}} f_{g, q}=-\mathcal{C}_{2 \leftrightarrow 2}[f]-\mathcal{C}_{1 \leftrightarrow 2}[f] .
$$

We include elastic $2 \leftrightarrow 2$ and colinear $1 \leftrightarrow 2$ collision processes at leading order in the coupling constant $\lambda=4 \pi \alpha_{s} N_{c}$. We consider $N_{c}=3$ colors and $N_{f}=3$ flavours of massless fermions with equal quark-antiquark content, i.e., at vanishing baryon chemical potential $\mu_{B}=0$. For the explicit expressions of collision kernels and the regularization procedure of the infrared divergences see the published work [7.

Following the previous work [11, initially at $\tau=1 Q_{s}^{-1}$ the fermion distribution function $f_{q}=0$ is set to zero, while the initial gluon density is

$$
f_{0}=\frac{2 A}{\lambda} \frac{Q_{0}}{\sqrt{p_{\perp}^{2}+p_{z}^{2} \xi^{2}}} e^{-\frac{2}{3} \frac{p_{\perp}^{2}+\xi^{2} p_{z}^{2}}{Q_{0}^{2}}},
$$

where the values of $A=5.24$ and $Q_{0}=1.8 Q_{s}$ are adjusted to match transverse momentum and energy density extracted from the lattice. The parameter $\xi$ determines the initial anisotropy and is set to $\xi=10$ to reflect highly anisotropic initial conditions in heavy ion collisions. We then follow the evolution of quark and gluon distribution functions to equilibrium at different values of the coupling constant $\lambda$ and find the corresponding values of shear viscosity over entropy ratio $\eta / s$. In the following we discuss the time evolution of energy density obtained by momentum integration of the non-equilibrium quark and gluon distribution functions.

It was observed in previous works [11/12 13]14], that the parametrically large differences in the equilibration rates for different coupling constants $\lambda$ can be largely scaled out by measuring time in units of relaxation time

$$
\tau_{R}(\tau)=\frac{4 \pi \eta / s}{T_{\mathrm{id} .}(\tau)}
$$

where $\eta / s$ is the specific shear viscosity. Note that for expanding system the local thermalization temperature and the relaxation time itself is time dependent. Here we define the effective temperature $T_{\mathrm{id}}(\tau)=\frac{\left.\left(T(\tau) \tau^{1 / 3}\right)\right|_{\tau \rightarrow \infty}}{\tau^{1 / 3}}$, which asymptotically coincides with the temperature of near equilibrium QGP.

In chemical equilibrium, $N_{f}=3$ fermions constitute $e_{q \text {,eq }} / e_{\text {total }} \approx 0.66$ of the total equilibrium energy density. In Fig. 1(a) we present the time evolution of quark energy density relative to equilibrium expectation as a function of rescaled time time $\tau / \tau_{R}$. We consider different values of the coupling constant $\lambda=1,5,10,20$, which corresponds to $\eta / s \approx 1900,2.8,1.0,0.4$ and vastly different relaxation times $\tau_{R}$. Remarkably, the large separation of equilibration time-scales 

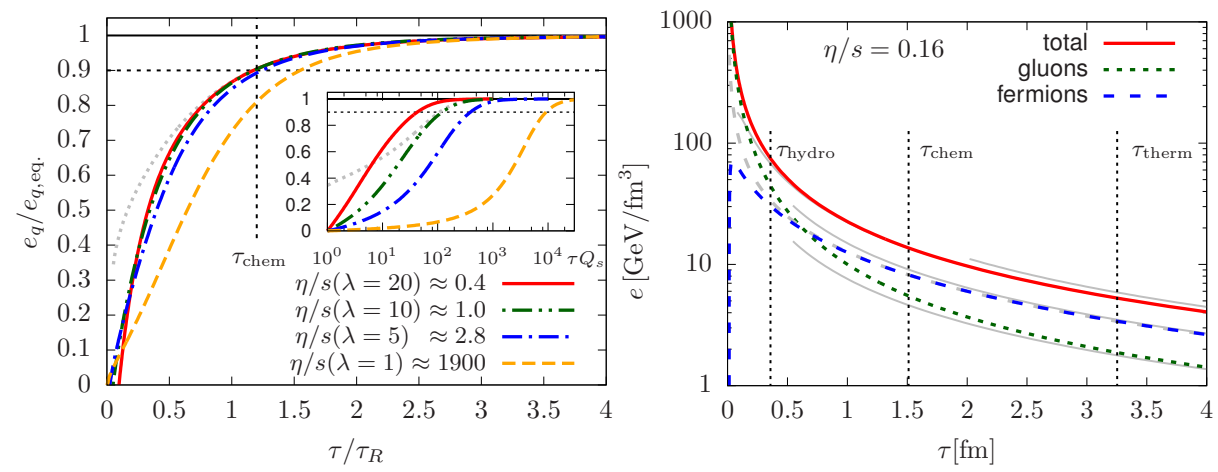

Fig. 1. a) Fermion energy density fraction $e_{q}(\tau) / e_{q}$,eq $(\tau)$ as a function of rescaled time for different coupling constants. The inset shows un-rescaled time dependence. b) Evolution of total energy density and its gluonic and fermion components in kinetic theory converted to physical units using universality of $\tau / \tau_{R}$ scaling and phenomenological values of $\eta / s=0.16,\langle s \tau\rangle=4.1 \mathrm{GeV}^{2}$ and $\nu_{\mathrm{eff}}=40$. The grey lines correspond to ideal, viscous and chemically equilibrated energies. Figures taken from Ref. $\underline{6}$

shown in the inset of Fig. 1 (a) collapses to near $\lambda$-independent equilibration curve shown in the main panel of Fig. 11(a). We find that at the time $\tau / \tau_{R} \approx 1.2$ and for larger values of the coupling, the fermions reach $90 \%$ of their chemical equilibrium energy density. We checked that this is not affected my small nonzero fermion density at early times indicated by grey dashed line in Fig. 11(a).

We can convert the scaled chemical equilibration time $\tau_{\text {chem }} / \tau_{R}$ to physical time by solving Eq. (3) and noting that the asymptotic constant $T_{\text {id. }}^{3} \tau=\left(T^{3} \tau\right)_{\infty}$ is proportional to the local entropy density per rapidity $(s \tau)_{\infty}$ :

$$
\tau_{\text {chem }}=\underbrace{\left(\tau_{\text {chem }} / \tau_{R}\right)^{3 / 2}}_{\text {scaled time variable }} \times \underbrace{(4 \pi \eta / s)^{3 / 2} \times(s \tau)_{\infty}^{-1 / 2} \times\left(4 \pi^{2} \nu_{\text {eff }} / 90\right)^{1 / 2}}_{\text {phenomenological input }} .
$$

Substituting the empirical estimate $\tau_{\text {chem }} / \tau_{R}=1.2$ we obtain a pocket formula for chemical equilibration time as a function of entropy density, specific shear viscosity and number of degrees of freedom. It is important to emphasize that although the parametric dependence of equilibration time on, say, entropy density, can be inferred from general physics arguments, Eq. (4) has all relevant numerical factors and is therefore a quantitative formula. The approximate independence of $\eta / s$ seen in Fig. 1(a), gives us motivation of plugging in phenomenological relevant parameter values of $\eta / s=2 / 4 \pi \approx 0.16,\langle s \tau\rangle=4.1 \mathrm{GeV}^{2}$ and $\nu_{\text {eff }}=40$. In Fig. 1 (b) we show the converted time evolution of total, gluon and fermion energy density. We observe that at $\tau \approx 0.6 \mathrm{fm}$ fermion and gluon energy contributions become equal and at $\tau_{\text {chem }} \approx 1.5 \mathrm{fm}$ the chemical equilibration defined above is achieved. In the paper [6]7] we also studied hydrodynamic and kinetic equilibrations and found the following ordering of equilibration scales 
(also shown in Fig. 1 (b))

$$
\underbrace{\tau_{\text {hydro }}}_{ \pm 10 \% \text { viscous } e(\tau)}<\underbrace{\tau_{\text {chem }}}_{ \pm 10 \% \text { fermion eq. } e(\tau)}<\underbrace{\tau_{\text {therm }}}_{ \pm 10 \% \text { ideal } e(\tau)} .
$$

Strikingly, the empirical weak coupling equilibration formula Eq. (4) produces realistic equilibration timescales compatible with heavy-ion phenomenology if extrapolated to realistic values of $\eta / s$. To what extent such extrapolation captures the dynamics of relatively strongly coupled system is debatable, but it is clear that the weak coupling equilibration baseline is not in contradiction with rapid thermalization of QGP.

Finally, Eq. (4) can be reformulated as a bound on charged particle multiplicities necessary to achieve chemical equilibrium by freeze-out. We relate the multiplicity $d N_{\mathrm{ch}} / d \eta$ in terms of scaled variables using $\langle\tau s\rangle \approx\left(S / N_{\mathrm{ch}}\right) 1 /\left(\pi R^{2}\right) d N_{\mathrm{ch}} / d \eta$ (where $S / N_{\mathrm{ch}} \approx 7$ [15]) and Eq. (40), so that

$$
\frac{d N_{\mathrm{ch}}}{d \eta}=\frac{4 \pi^{3}}{90} \nu_{\mathrm{eff}}\left(S / N_{\mathrm{ch}}\right)^{-1}(4 \pi \eta / s)^{3}\left(\tau / \tau_{R}\right)^{3}(\tau / R)^{-2} .
$$

Assuming that the system disintegrates once its lifetime exceeds the system size $\tau \sim R$ [14, the minimum multiplicity needed for that time to be at or above chemical equilibration time $\tau_{\text {chem }} / \tau_{R}=1.2$ is

$$
\frac{d N_{\mathrm{ch}}}{d \eta} \gtrsim 110\left(\frac{\tau_{\text {chem }}}{1.2 \tau_{R}}\right)^{3}\left(\frac{\eta / s}{0.16}\right)^{3}\left(\frac{\tau_{\text {chem }}}{R}\right)^{-2} .
$$

This bound is roughly compatible with experimentally observed trends of strangeness enhancement [1/2]3, which gives theoretical ground for the assumed formation of chemically equilibrated QGP at high multiplicity collisions.

In summary, we presented the detailed picture of QGP equilibration within QCD kinetic theory framework. Strikingly, extrapolated to phenomenological values of $\eta / s$, this picture gives realistic equilibration timescales and connects in a novel way the transport properties of QGP to experimental observations of hadron chemistry in proton-proton, proton-nucleus and nucleus-nucleus collisions.

This work was supported in part by the German Research Foundation (DFG) Collaborative Research Centre (SFB) 1225 (ISOQUANT) (A.M.).

\section{References}

1. Adam, J., et al.: Enhanced production of multi-strange hadrons in high-multiplicity proton-proton collisions. Nature Phys. 13, 535-539 (2017). DOI 10.1038/ nphys4111

2. Abelev, B.B., et al.: Multiplicity Dependence of Pion, Kaon, Proton and Lambda Production in $\mathrm{p}-\mathrm{Pb}$ Collisions at $\sqrt{s_{N N}}=5.02 \mathrm{TeV}$. Phys. Lett. B728, 25-38 (2014). DOI 10.1016/j.physletb.2013.11.020 
3. Abelev, B.B., et al.: Multi-strange baryon production at mid-rapidity in $\mathrm{Pb}-\mathrm{Pb}$ collisions at $\sqrt{s_{N N}}=2.76 \mathrm{TeV}$. Phys. Lett. B728, 216-227 (2014). DOI 10.1016/j.physletb.2014.05.052,10.1016/j.physletb.2013.11.048. [Erratum: Phys. Lett.B734,409(2014)]

4. Andronic, A., Braun-Munzinger, P., Redlich, K., Stachel, J.: Decoding the phase structure of QCD via particle production at high energy. Nature 561(7723), 321330 (2018). DOI 10.1038/s41586-018-0491-6

5. Schlichting, S., Teaney, D.: The First fm/c of Heavy-Ion Collisions (2019)

6. Kurkela, A., Mazeliauskas, A.: Chemical equilibration in hadronic collisions. Phys. Rev. Lett. 122, 142,301 (2019). DOI 10.1103/PhysRevLett.122.142301

7. Kurkela, A., Mazeliauskas, A.: Chemical equilibration in weakly coupled QCD. Phys. Rev. D99(5), 054,018 (2019). DOI 10.1103/PhysRevD.99.054018

8. Arnold, P.B., Moore, G.D., Yaffe, L.G.: Effective kinetic theory for high temperature gauge theories. JHEP 01, 030 (2003). DOI 10.1088/1126-6708/2003/01/030

9. Baier, R., Mueller, A.H., Schiff, D., Son, D.T.: 'Bottom up' thermalization in heavy ion collisions. Phys. Lett. B502, 51-58 (2001). DOI 10.1016/S0370-2693(01) 00191-5

10. Berges, J., Boguslavski, K., Schlichting, S., Venugopalan, R.: Turbulent thermalization process in heavy-ion collisions at ultrarelativistic energies. Phys. Rev. D89(7), 074,011 (2014). DOI 10.1103/PhysRevD.89.074011

11. Kurkela, A., Zhu, Y.: Isotropization and hydrodynamization in weakly coupled heavy-ion collisions. Phys. Rev. Lett. 115(18), 182,301 (2015). DOI 10.1103/ PhysRevLett.115.182301

12. Keegan, L., Kurkela, A., Romatschke, P., van der Schee, W., Zhu, Y.: Weak and strong coupling equilibration in nonabelian gauge theories. JHEP 04, 031 (2016). DOI 10.1007/JHEP04(2016)031

13. Kurkela, A., Mazeliauskas, A., Paquet, J.F., Schlichting, S., Teaney, D.: Effective kinetic description of event-by-event pre-equilibrium dynamics in high-energy heavy-ion collisions. Phys. Rev. C99(3), 034,910 (2019). DOI 10.1103/PhysRevC. 99.034910

14. Kurkela, A., Mazeliauskas, A., Paquet, J.F., Schlichting, S., Teaney, D.: Matching the Nonequilibrium Initial Stage of Heavy Ion Collisions to Hydrodynamics with QCD Kinetic Theory. Phys. Rev. Lett. 122(12), 122,302 (2019). DOI 10.1103/ PhysRevLett.122.122302

15. Hanus, P., Mazeliauskas, A., Reygers, K.: Entropy production in pp and $\mathrm{Pb}-\mathrm{Pb}$ collisions at the LHC (2019) 